\title{
Mechanical and Dielectric Spectroscopy of Aroclor, 1,2-Polybutadiene, and Their Mixtures
}

\author{
C. M. Roland
}

Naval Research Laboratory, Chemistry Division, Code 6120, Washington, D.C. 20375-5342

Received November 7, 1994; Revised Manuscript Received January 26, $1995^{\circ}$

\begin{abstract}
Dynamic mechanical and dielectric spectra were obtained in the glass transition zone for Aroclor 1248 (a polychlorinated biphenyl), for a low molecular weight polybutadiene $(88 \% 1,2-$ microstructure), and for a 50\% mixture. The neat materials exhibit two different and unusual characteristics. For Aroclor, the shape of the relaxation function measured mechanically is quite different from the dielectric result. In accord with the well-established correlation between time and temperature dependences, the relaxation times for the (narrower) dielectric spectrum exhibit a weaker temperature dependence. Dielectric and mechanical relaxation functions measured for the polybutadiene are in close agreement; however, the shape of the spectrum varies with temperature. Thus, neat polybutadiene with high vinyl content is thermorheologically complex at temperatures near $T_{\mathrm{g}}$. The $50 \%$ mixture displays the previously observed anomaly of isothermal relaxation proceeding faster than that of the neat components. A comparison of this effect as measured mechanically and dielectrically implies that its origin is a positive excess mixing volume.
\end{abstract}

\section{Introduction}

Mixing changes the time scale of the segmental motions in polymers and the reorientational dynamics of small molecules. ${ }^{1-3}$ Most interesting are mixtures whose relaxation behavior is not intermediate between that of the pure components. This anomaly, which has been observed in polymer/diluent systems and in polymer blends, has an uncertain origin. Two mechanisms have been proposed. One hypothesis ${ }^{3-5}$ is that an excess mixing volume, and consequent change in unoccupied volume, underlies mixture relaxation that is faster or slower than either neat component. A second suggested mechanism, ${ }^{6}$ drawn from the coupling model of relaxation, ${ }^{7,8}$ is based on the idea that the glass transition temperature, as well as the magnitude of the relaxation time measured near $T_{\mathrm{g}}$, is greatly amplified by intermolecular cooperativity; that is, interferences from neighboring segments slow down the local motions. This means that intermolecular cooperativity governs to a significant extent the magnitude of the observed relaxation times. When two components are mixed, dilution markedly alters the nature of this intermolecular cooperativity. Accordingly, the relaxation time of a neat component relevant for deducing the effect of mixing is not the relaxation time (or $T_{\mathrm{g}}$ ) actually measured for the neat liquid. Rather, a much shorter noncooperative relaxation time is the appropriate quantity to assess. This noncooperative relaxation time can be deduced from the measured relaxation time and the associated relaxation function. ${ }^{6-8}$

One example of the unusual mixture dynamics is found in the miscible blend of polychloroprene and epoxidized polyisoprene. ${ }^{5}$ When the epoxidation of the latter is $25 \mathrm{~mol} \%$, the two polymers have virtually the same glass transition temperature and, at least for temperatures near $T_{\mathrm{g}}$, equivalent segmental relaxation times. ${ }^{9,10}$ However, their blends exhibit relaxation times as much as an order of magnitude shorter than either neat polymer, a result ascribed to the large density decrease (and thus positive excess volume) accompanying mixing. ${ }^{5}$

The opposite anomaly has been found in blends of poly(vinyl methyl ether) (PVME) and poly(epichlorohy-

${ }^{\otimes}$ Abstract published in Advance ACS Abstracts, March 15, 1995 drin) (PECH). Despite the close equivalence of the polymers' glass transition temperatures and segmental relaxation times, their blends relax slower than either neat component. 11,12 The origin of this behavior has yet to be fully investigated.

The small-molecule glass former 1,1-bis( $p$-methoxyphenyl)cyclohexane (BMC) has a lower $T_{\mathrm{g}}$ than poly(methylphenylsiloxane) (PMPS) when the latter's molecular weight is high (ca. 100000 ). Nevertheless, mixing with PMPS speeds up relaxation of BMC. ${ }^{13,14}$ Since addition of the polymer increases the density, the only viable explanation for the anomaly in this system is a change in intermolecular cooperativity. Although this cannot be accessed merely from consideration of the neat components' $T_{\mathrm{g}}$ 's, it can be qualitatively predicted from the coupling model of relaxation..$^{13}$

A final example of anomalous relaxation in mixtures is the one with which the present paper is concerned. The reorientational mobility of Aroclor (tradename for a polychlorinated biphenyl) increases upon addition of 1,2-polybutadiene, even though the latter has a higher glass transition temperature ${ }^{4,6}$ From the published work to date, it is not clear which of the proposed mechanisms gives rise to the phenomenon in this mixture. Since the phenomenon of relaxation dynamics in a mixture not being intermediate to that of the neat components is likely to be found in more than a few isolated systems, and moreover reflects interesting new physics, the purpose herein was to assess the proposed hypotheses and determine if either is the likely source of the relaxation behavior in $\mathrm{PBD} /$ Aroclor mixtures.

\section{Experimental Section}

The polybutadiene (Nisso B2000 from Nippon Soda Co.) had a molecular weight of 2000 and was measured by NMR to have a vinyl content of $88 \%$. Aroclor 1248 (Monsanto Co.) is a mixture of chlorinated biphenyls having an average chlorine content of $48 \%$. A mixture was prepared of equal weights of the two viscous liquids.

Dynamic mechanical measurements were obtained with a Bohlin VOR rheometer. A parallel-plate geometry was used, with sample radii and gaps of typically 3 and $2 \mathrm{~mm}$, respectively. Dielectric experiments employed an Imass, Inc., time domain spectrometer. Samples were contained between $\mathrm{Al}$ 


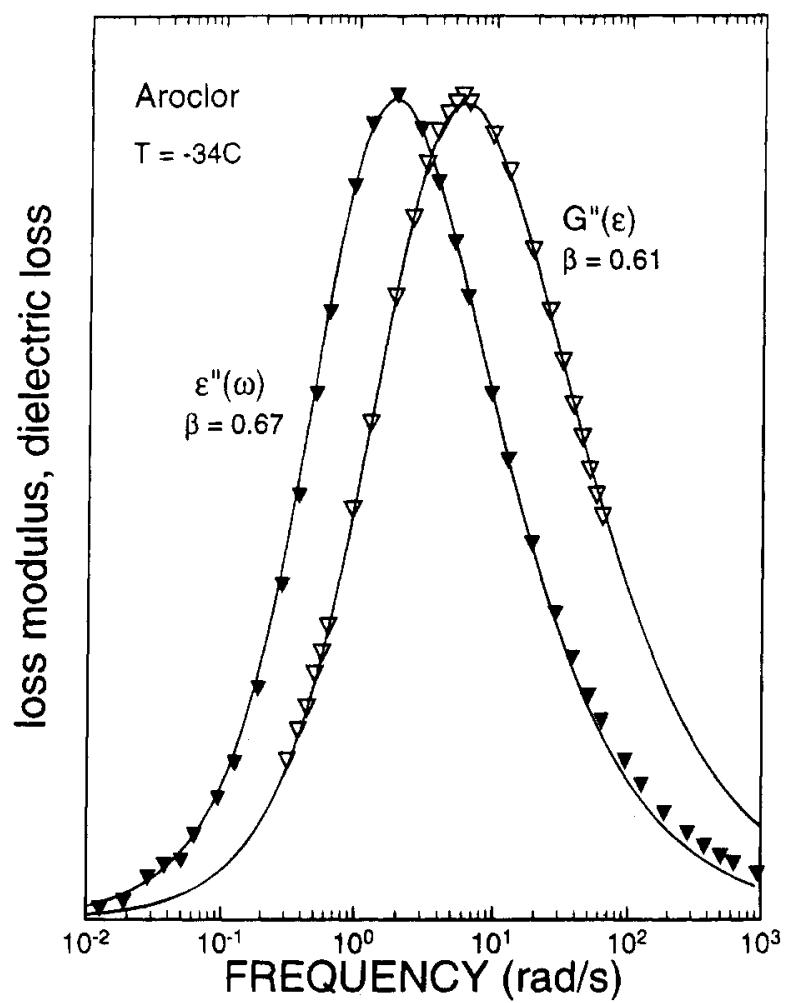

Figure 1. Glass transition dispersion in the dielectric (filled symbols) and dynamic mechanical (hollow symbols) spectra measured for Aroclor 1248 at $-34^{\circ} \mathrm{C}$. The loss modulus peak is broader, as reflected in the smaller stretch exponent (eq 2) and is shifted to higher frequencies than the dielectric loss.

plates ( $c a .25 \mathrm{~m}$ radius with a $0.1 \mathrm{~mm}$ gap), whose separation was maintained with Teflon spacers. For both instruments, sample temperatures were maintained constant to within \pm 0.1 ${ }^{\circ} \mathrm{C}$.

\section{Results and Discussion}

3.1. Neat Liquids. Representative loss modulus and dielectric loss spectra of Aroclor are displayed in Figure 1. These data can be adequately described by the well-known stretched-exponential function, ${ }^{15,16}$ which for the mechanical loss modulus is

$$
G^{\prime \prime}(\omega)=\omega \int_{0}^{\infty} G(t) \cos (\omega t) \mathrm{d} t
$$

with

$$
G(t)=G_{0} \exp \left[-(t / \tau)^{-\beta}\right]
$$

In eq $2 G_{0}$ is the unrelaxed modulus, $\tau$ is the segmental relaxation time, and the stretch exponent has a value in the range $0 \leq \beta \leq 1$. The equations for the dielectric loss, $\epsilon^{\prime \prime}(\omega)$, have the same form, with the dielectric constant replacing the relaxation modulus. The relaxation times obtained by fitting the experimental spectra are shown in Figure 2. For Aroclor no dependence of $\beta$ on temperature was observed. The interesting result is that the stretch exponent is found to be significantly smaller for mechanical data $(\beta=0.60 \pm 0.01)$ than for dielectric measurements $(\beta=0.68 \pm 0.01)$. These results bracket the value, $\beta=0.63$, determined previously by dynamic light scattering. ${ }^{17}$ Thus, Aroclor exhibits an unusual dependence of its relaxation dynamics on the experimental technique employed for measurements. While relaxation times often vary with experimental probe, only for a very few polymers has $\beta$

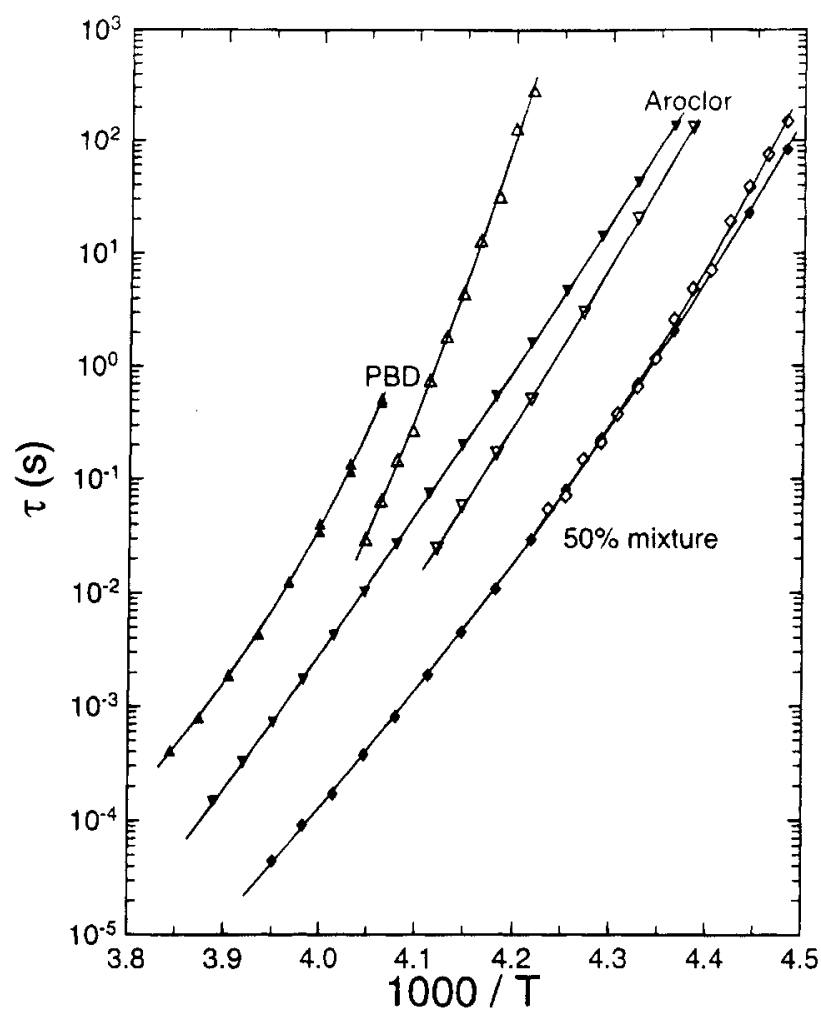

Figure 2. Dielectric (filled symbols) and dynamic mechanical (hollow symbols) relaxation times measured at various temperatures near $T_{g}$ for $88 \%$ vinyl PBD $(\triangle, \Delta)$, Aroclor $1248(\nabla, \nabla)$, and a $50 \%$ by weight mixture $(\diamond, \diamond)$. The data for the mixture are displaced toward shorter times (lower temperatures). The lines through the data points represent the fits to eq 3 .

been found to depend on the measurement technique. ${ }^{18-20}$ For polymers, a probe dependence in the shape of the relaxation function can arise from a polar side group dominating the dielectric response, with the mechanical behavior reflecting primarily backbone motions. Previous results on small-molecule liquids, in which local relaxation entails primarily reorientational motion, found the stretch exponent to be independent of the method of measurement. ${ }^{21}$

The magnitude of $\beta$ has been shown for many polymers and glass-forming liquids to be correlated with the sensitivity of the relaxation times to temperature. ${ }^{21-35}$ Smaller values of $\beta$ correspond to relaxation times that change more with changes in temperature. This correlation has been rationalized in terms of a liquid's local structure and how the density of available configurations changes with temperature. ${ }^{31,32}$ The relationship between time and temperature dependencies means that the ratio of the quantities $B / T_{\infty}$ from the VogelFulcher equation ${ }^{1}$

$$
\tau^{*}=A \exp \left(B /\left(T-T_{\infty}\right)\right)
$$

increases with increasing $\beta .23,24$

The correlation, which can be derived ${ }^{36}$ from extension of the Adam-Gibbs theory ${ }^{37}$ of glass-forming liquids, is predicted by the coupling model of relaxation. Specifically, at high temperatures and for relaxations characterized by large values of $\beta$ (whereby eq 3 assumes an Arrhenius form), the product of the measured activation energy and the stretch exponent, $\beta$, is anticipated to be a constant, equal to the activation energy in the absence of intermolecular cooperativity. ${ }^{38}$ The Arrhenius plots of the Aroclor relaxation times are 
Table 1. Relative Magnitude of the Noncooperative Relaxation Times As Measured Dielectrically and Mechanically $\left(T=-28^{\circ} \mathrm{C}\right)$

\begin{tabular}{lllll}
\hline & & \multicolumn{1}{c}{$\beta$} & \multicolumn{1}{c}{$\tau$} & \multicolumn{1}{c}{$\tau_{0}$} \\
\hline Aroclor & $\epsilon^{\prime \prime}(\omega)$ & 0.69 & $2.9 \times 10^{-2}$ & $3.0 \times 10^{-5}$ \\
PBD & $\epsilon^{\prime \prime}(\omega)$ & 0.39 & 1.2 & $2.0 \times 10^{-7}$ \\
Aroclor & $G^{\prime \prime}(\omega)$ & 0.60 & $7.2 \times 10^{-3}$ & $1.8 \times 10^{-6}$ \\
PBD & $G^{\prime \prime}(\omega)$ & 0.39 & 0.15 & $8.9 \times 10^{-8}$
\end{tabular}

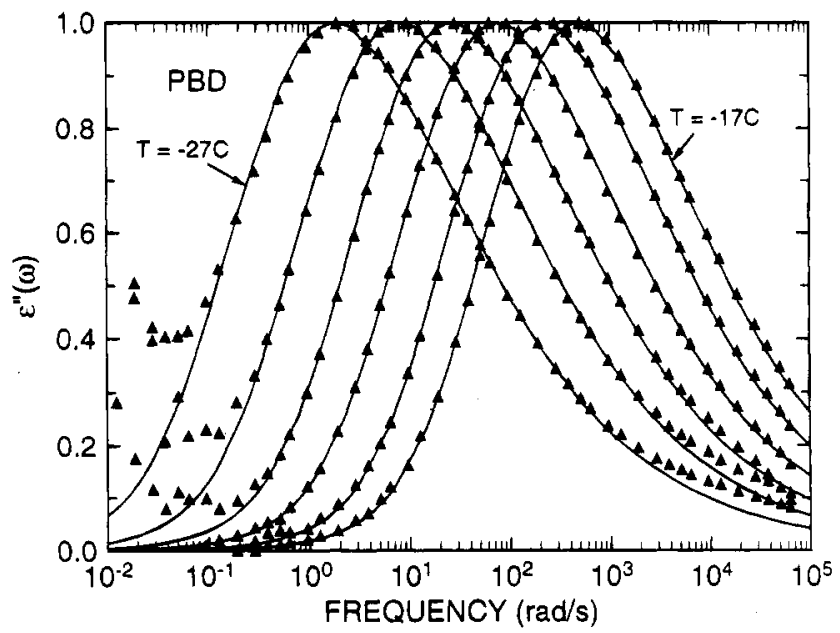

Figure 3. Isothermal segmental relaxation peak in the dielectric loss of PBD displayed at $2{ }^{\circ} \mathrm{C}$ increments from -27 to $-17^{\circ} \mathrm{C}$. The peak broadens with decreasing temperature (e.g., best fit $\beta=0.43$ at the highest and 0.39 at the lowest temperature).

nearly linear (Figure 2), with activation energies calculated to be 137 and $150 \mathrm{kcal} / \mathrm{mol}$ for the dielectric and mechanical data, respectively (the exact values are temperature dependent, since the curves in Figure 2 are not exactly linear). The ratio of these activation energies is nearly equal to the ratio of the stretch exponents measured by the two spectroscopies (Table 1 and Figure 1), which differ by $15 \%$.

For the few polymer cases in which $\beta$ has been found to vary with measurement technique, the correlation between the value of the stretch exponent and the temperature sensitivity of $\tau$ is still maintained. As shown in Figure 2, this is also the case for Aroclor. Its mechanical relaxation time varies more with changes in temperature than does the value of $\tau$ extracted from the dielectric data. Thus, while Aroclor exhibits an unusual probe dependence for $\beta$, the correlation between time and temperature dependencies is maintained, even when different dynamical variables (e.g., dipole moment versus modulus) are used to probe the local dynamics.

The behavior of PBD is quite different. In agreement with prior results on a high molecular weight 1,2polybutadiene, ${ }^{20}$ a comparison of the mechanical and dielectric results yields the following observations: (i) The stretch exponent, when compared at the same temperature, is identical for the two spectroscopies. (ii) However, as illustrated in Figure $3, \beta$ varies with temperature, a $10^{\circ} \mathrm{C}$ increase in temperature increasing $\beta$ by about 0.04 . This effect has been seen in poly(vinyl acetate) as well. ${ }^{39}$ (iii) Notwithstanding equivalence of the $\beta$, at any given temperature, the dielectric relaxation times are longer (by roughly a factor of 5) than the mechanical relaxation times (see Figure 2). This is unsurprising, since different probes of local motion involve different dynamical variables and different correlation functions. ${ }^{40,41}$ The time-temperature shift factors are the same for the two spectroscopies, as expected, since the $\beta$ 's are very similar.

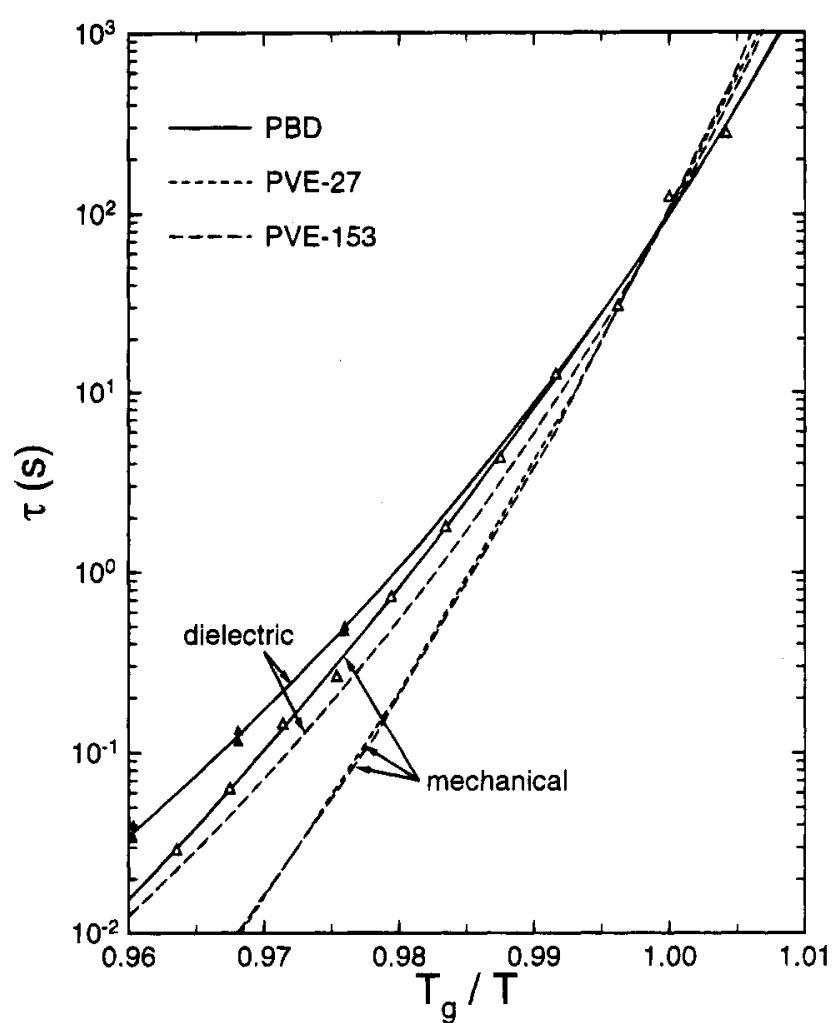

Figure 4. $T_{\mathrm{g}}$-normalized Arrhenius plots of segmental relaxation times for PBD (mechanical and dielectric data), for a 27000 molecular weight 1,2-polybutadiene (mechanical data), and for a 153000 molecular weight 1,2-polybutadiene (mechanical and dielectric data). A steeper cooperativity curve, reflecting more intermolecularly cooperative motion, is associated with a smaller value of $\beta$ (eq 2 ).

The Arrhenius plots for the Aroclor in Figure 2 are almost linear, allowing a direct comparison of slopes (i.e., activation energies). However, polymer segmental relaxation times usually have a non-Arrhenius temperature dependence; thus, some normalization scheme must be used to compare the temperature sensitivity. It has been amply demonstrated that the fragility plot (or, using more appropriate terminology, the cooperativity curve) is a self-consistent means to classify and distinguish segmental relaxation behavior. ${ }^{25,26,29} \mathrm{~A}$ cooperativity curve is simply an Arrhenius plot of the relaxation times with temperature normalized by the glass transition temperature. ${ }^{29,31,32}$

$T_{\mathrm{g}}$-scaled Arrhenius plots of the mechanical and dielectric results for PBD are shown in Figure 4. Since both spectroscopies yield the same relaxation function (same $\beta$ ), we expect the temperature dependence of the measured relaxation time to be the same. However, the relaxation times differ; a change of the relaxation time by a multiplicative factor introduces a spurious (in that it does not reflect intermolecular cooperativity) change in the cooperativity curve. This is primarily because the normalization variable, $T_{\mathrm{g}}$, is taken to be the temperature at which the relaxation time assumes an arbitrary value ( $100 \mathrm{~s}$ herein). With the glass transition operationally defined in this manner, the mechanical data, having a shorter $\tau$, will automatically appear to be more cooperative (steeper curve). This is indeed observed in Figure 4.

Also shown in Figure 4 are literature data $a^{4,35}$ for two higher molecular weight, higher vinyl (ca. 95\%) polybutadienes, PVE-27 and PVE-153, having molecular weights of 27000 and 153000 , respectively. PVE refers 
to poly(vinylethylene), the structure-based name for $1,2-$ polybutadiene. Both these polymers exhibit the same temperature dependence of $\tau$, which is somewhat stronger than that found for PBD. It is anticipated that polymers differing only in molecular weight, while having different $T_{\mathrm{g}}$ 's and $\tau$ 's, will exhibit equivalent cooperativity plots. This is because chain length per se does not modify the segmental relaxation function. ${ }^{29}$ From previous work, ${ }^{27}$ we expect that due to its lower vinyl content, segmental relaxation of $\mathrm{PBD}$ will be less intermolecularly cooperative. Interestingly, its stretch exponent is roughly the same as those for the higher vinyl polybutadienes, notwithstanding its less steep cooperativity curve.

3.2. Mixtures. The relaxation properties of 1,2 polybutadiene/Aroclor mixtures are of particular interest because of the anomaly observed therein. Both depolarized light scattering measurements ${ }^{6}$ and $d y-$ namic mechanical spectroscopy ${ }^{4}$ demonstrate that the presence of the polymer decreases the reorientational relaxation time of the Aroclor, an unexpected result given that the former's $T_{\mathrm{g}}$ is significantly higher than that of neat Aroclor. As described above, two hypotheses have been suggested to explain this anomaly. The more obvious is that mixing increases the unoccupied volume, speeding up the local motions. In fact, the density of 1,2-polybutadiene/Aroclor mixtures is less than the linear interpolation of the pure components' densities, ${ }^{4}$ indicating a positive excess volume. The second explanation is based on the coupling model of relaxation $^{6-8}$ wherein the observed relaxation time, which is strongly dependent on intermolecular interactions, is related to a "noncooperative" relaxation time, $\tau_{0}$, according to

$$
\tau=\left[\beta t_{\mathrm{c}}^{\beta-1} \tau_{0}\right]^{1 / \beta}
$$

This noncooperative relaxation time, $\tau_{0}$, prevails at short times $\left(t<t_{\mathrm{c}}\right)$ before torques and unbalanced forces from neighboring segments have built up to an extent sufficient to impede relaxation. Equation 4 is obtained by recognizing that at the crossover time, $t_{c}$, the relaxation function describing noncooperative relaxation ("Debye" relaxation) must be equal to the relaxation function given in eq 2. (Note that an earlier derivation, ${ }^{7}$ assuming continuity of the relaxation rate rather than the relaxation function, led to a slightly different form for eq 4.) $\tau_{0}$ reflects the local friction, and because of the nonlinear relationship between $\tau$ and $\tau_{0}$, there can exist a reversal in the relative magnitude of the observed $(\tau)$ and the noncooperative $\left(\tau_{0}\right)$ relaxation times for two liquids. Particularly when their relaxations are characterized by widely different $\beta$ 's, this leads to the possibility that addition of a neat component of higher $T_{\mathrm{g}}$ (and thus longer $\tau$ ) can speed up the relaxation.

Taking for the crossover time $t_{\mathrm{c}}=2 \times 10^{-12} \mathrm{~s},{ }^{42}$ we can calculate from eq 4 the noncooperative relaxation times of PBD and Aroclor, as determined both dielectrically and mechanically. To make the comparison at the same temperature, we use the fitted Vogel-Fulcher equation (eq 3 ) to interpolate the measured data. The results are given in Table 1 , where it is seen that, as measured dielectrically, the noncooperative relaxation time of PBD is 150 times shorter than that of Aroclor. Hence, addition of PBD should reduce the local friction, consistent with faster relaxation in the mixture. This reversal in relaxation time (cooperative to noncooperative) is also present, but to a lesser degree, in the mechanical data (see Figure 5). Due to the smaller

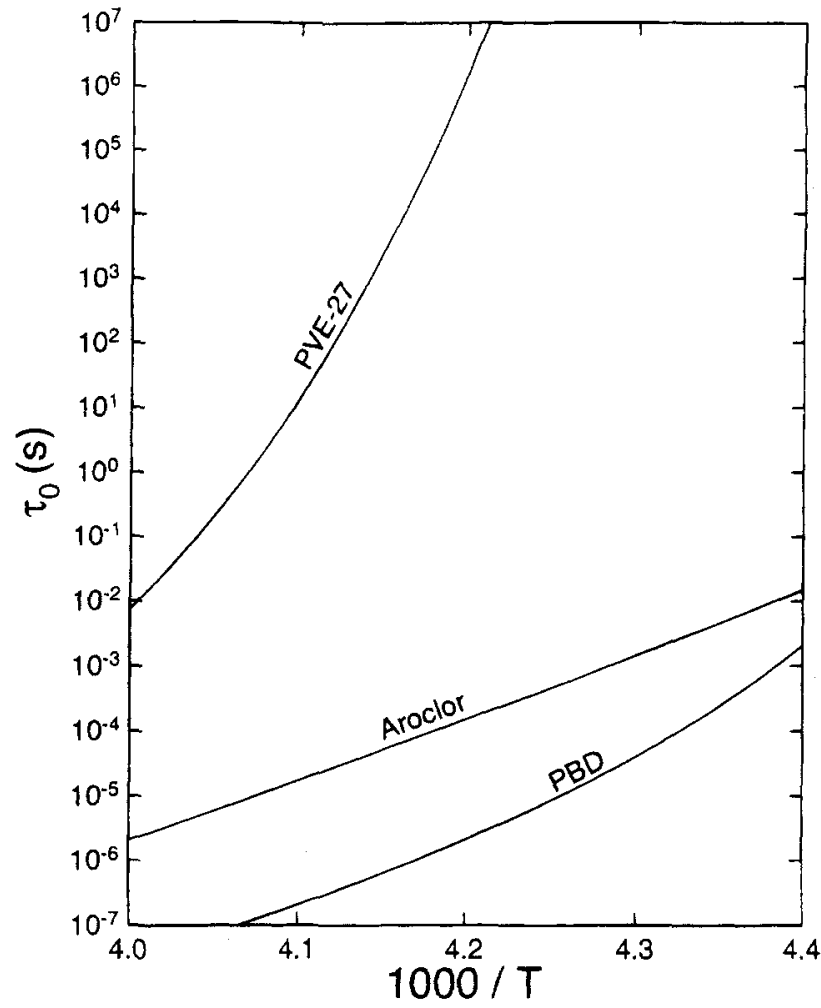

Figure 5. Noncooperative relaxation time calculated using eq 4 and the fit of eq 3 to dynamical mechanical loss modulus results. The data for Aroclor and PVE-27 are taken from ref 4. Note that, even though the measured relaxation times of PBD are longer than those of Aroclor, there is a reversal in the relative magnitude for $\tau_{0}$. This is not the case for Aroclor and PVE-27, although their mixtures still show the anomalous speeding up of the local dynamics.

difference in the components' $\beta$ 's, however, the $\tau_{0}$ 's differ by only a factor of 20 (Table 1 ). If the mechanism suggested by the coupling model does indeed explain the anomalous speeding up of the mixture's relaxation, then the anomaly should be manifested substantially more in the dielectric data.

In Figure 2 are displayed the dielectric and the mechanical relaxation times for the 50/50 mixture. The results for the two measurement techniques are almost equal, despite the order of magnitude disparity in the ratio of the noncooperative relaxation times of the components as measured dielectrically versus mechanically. It is important to note that the greater polarity of Aroclor means that it contributes more than PBD to the dielectric response of the 50/50 mixture. Presumably, the mechanical data reflect the contribution of both components more evenly. This effect alone causes the dielectric data for the mixture to be weighted more toward shorter times. Nevertheless, the dielectric data for the mixture do not lie toward lower temperatures or shorter values of $\tau$. Clearly, the anomalous speeding up is not more evident as measured dielectrically than in the mechanical results. This is difficult to reconcile with the notion ${ }^{6}$ that the relative magnitude of the noncooperative relaxation times for the components is the dominant factor governing the mixture dynamics. The results in Figure 2 are consistent with the alternative viewpoint that the positive excess volume of mixing, and consequently greater unoccupied volume, underlies faster relaxation in the mixture. This effect would influence the mechanical and dielectric behaviors in a similar fashion. 


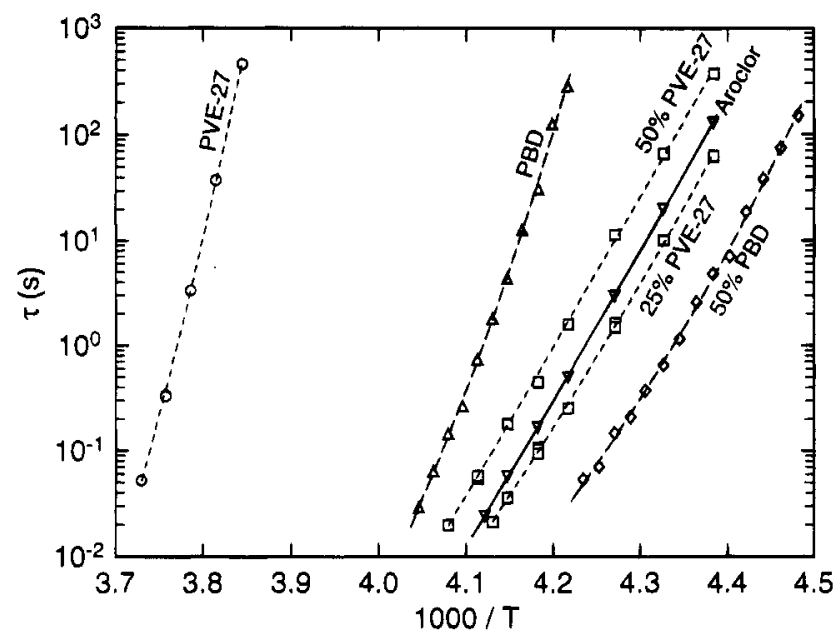

Figure 6. Segmental relaxation times obtained from dynamic mechanical measurements on $\operatorname{PBD}(\Delta)$, Aroclor $(\nabla)$, and an equal-weight mixture $(\diamond)$, the latter having shorter relaxation times than either neat component. A similar effect is seen in mixtures of Aroclor with PVE-27 (口), the $25 \%$ polymer mixture relaxing faster than pure Aroclor. The data for the neat PVE$27(0)$ fall at higher temperature, reflecting its higher $T_{\mathrm{g}}$.

If we compare (Figure 6) the data from Aroclor/PBD mixtures to the results for Aroclor with the high molecular weight PVE-27, we find that the relaxation times for both exhibit a peculiar composition dependence. At higher Aroclor concentrations, the Aroclor/ PVE-27 mixture has shorter relaxation times than neat Aroclor. ${ }^{4}$ However, the calculation from eq 4 actually predicts anomalous behavior only for the PBD mixtures, not for Aroclor with PVE-27 (see Figure 5). For Aroclor/ PVE-27, the anomaly of faster mixture relaxation ( $\left.\tau_{\text {mixture }}<\tau_{\text {Aroclor }}\right)$ is observed, notwithstanding the absence of the reversal $\left(\tau_{\mathrm{PVE}} / \tau_{\text {Aroclor }}>1 ; \tau_{0, \mathrm{PVE}} / \tau_{0, \text { Aroclor }}\right.$ $<1$ ) in magnitude of the relaxation times (cf. Figures 5 and 6). Thus, only the hypothesis based on the increase in unoccupied volume is consistent with the fact that both mixtures exhibit the anomaly.

\section{Summary}

PBD and Aroclor show disparate glass transition behaviors. For most glass-forming liquids, the shape of the relaxation function, as reflected in the value of the stretch exponent $\beta$, is invariant to temperature and to the experimental probe used for measurements. Exceptions to these properties are found for PBD (temperature dependence) and Aroclor (probe dependence). The fact that $\beta$ varies with temperature, together with the difference in relaxation time (but not shape) when measured mechanically versus dielectrically, complicates the interpretation of the polymer's cooperativity curve in terms of intermolecular cooperativity. Nevertheless, the correlation of time and temperature dependencies is maintained. This correlation also holds for Aroclor-the mechanical relaxation times change more with temperature than do the dielectric relaxation times, in accord with the broader relaxation function (smaller $\beta$ ) measured by dynamic mechanical spectroscopy.

The mixture of PVE and Aroclor displays the previously observed anomaly of having a relaxation time that is less than that of either neat component. The fact that the degree to which the mixture relaxes faster is not greatly different as measured by mechanical versus dielectric spectroscopy is consistent with the hypothesis that it is the result of the decrease in density, and hence greater unoccupied volume, accompanying mixing. At least for this particular system, no recourse is necessary to the mechanism based on the coupling model.

Acknowledgment. The author thanks the E. L. Puskas Co. for providing the low molecular weight polybutadiene and K. J. McGrath of NRL, who carried out the NMR determination of its vinyl content. This work was supported by the Office of Naval Research.

\section{References and Notes}

(1) Ferry, J. D. Viscoelastic Properties of Polymers; Wiley: New York, 1980

(2) Lodge, T. P. J. Phys. Chem. 1993, 97, 1480.

(3) Fujita, H. Macromolecules 1993, 26, 643, 4720.

(4) Santangelo, P. G.; Roland, C. M.; Ngai, K. L. Macromolecules $1994,27,3859$.

(5) Roland, C. M.; Santangelo, P. G.; Baram, Z.; Runt, J. Macromolecules 1994, 27, 5382 .

(6) Rizos, A.; Ngai, K. L. Phys. Rev. B 1992, 46, 8126.

(7) Ngai, K. L.; Rendell, R. W.; Rajagopal, A. K.; Teitler, S. Ann. N.Y. Acad. Sci. 1986, 484, 150.

(8) Ngai, K. L. In Disorder Effects on Relaxation ProcessesGlasses, Polymers, Proteins; Richert, R., Blumen, A., Eds.; Springer-Verlag: Berlin, 1994; pp 89-150.

(9) Walton, J. H.; Miller, J. B.; Roland, C. M. J. Polym. Sci. Polym. Phys. Ed. 1992, 30, 527.

(10) Nagode, J. B.; Roland, C. M. Polymer 1991, 32, 505.

(11) Alegria, A.; Telleria, I.; Colmenero, J. J. Non-Cryst. Solids 1994, 172-174, 961

(12) McGrath, K. J.; Roland, C. M. Rubber Chem. Technol, 1994, 67,805 .

(13) Roland, C. M.; Santangelo, P. G.; Ngai, K. L.; Meier, G. Macromolecules 1993, 26, 6164.

(14) Lohfink, M.; Sillescu, H. In Proceedings of the 1st Tohwa University International Symposium; Kawasaki, $\mathrm{K}$, Kawakatsu, T., Tokuyama, M., Eds.; American Institute of Physics: New York, 1992.

(15) Kohlrausch, R. Pogg. Ann. Phys. 1847, 12, 393.

(16) Williams, G.; Watts, D. C. Trans. Faraday Soc. 1970, 66, 80.

(17) Rizos, A.; Fytas, G.; Lodge, T. P.; Ngai, K. L. J. Chem. Phys. $1991,95,2980$.

(18) Ngai, K. L.; Mashimo, S.; Fytas, G. Macromolecules 1988, 21,3030 .

(19) Takeuchi, H.; Roe, R. J. J. Chem. Phys. 1992, 94, 7446.

(20) Colmenero, J.; Alegria, A.; Ngai, K. L.; Roland, C. M. Macromolecules 1994, 27, 407.

(21) Bohmer, R.; Ngai, K. L.; Angell, C. A.; Plazek, D. J. J. Chem. Phys. 1993, 99, 4201.

(22) Hodge, I. M. Macromolecules 1993, 16, 898; 1987, 20, 2897; J. Non-Cryst. Solids 1991, 131-133, 435.

(23) Tatsumisago, M.; Haltpap, B. L.; Green, J. L.; Lindsay, S. M.; Angell, C. A. Phys. Rev. Lett. 1990, 64, 1549.

(24) Sethna, J. P. Europhys. Lett. 1988, 6, 529.

(25) Bohmer, R.; Ngai, K. L.; Angell, C. A.; Plazek, D. J. J. Chem. Phys. 1993, 99, 4201.

(26) Plazek, D. J.; Ngai, K. L. Macromolecules 1991, 24, 1222.

(27) Roland, C. M.; Ngai, K. L. Macromolecules 1991, 24, 5315; $1992,25,1844$.

(28) Roland, C. M.; Ngai, K. L. Macromolecules 1992, 25, 363.

(29) Roland, C. M.; Ngai, K. L. Macromolecules 1992, 25, 5765.

(30) Roland, C. M. Macromolecules 1992, 25, 7031.

(31) Angell, C. A. In Relaxations in Complex Systems; Ngai, K. L., Wright, G. B., Eds.; Government Printing Office: Washington, DC, 1985 ; $\mathrm{p} 3$.

(32) Angell, C. A. J. Non-Cryst. Solids 1991, 131-133, 13.

(33) Torell, L. M.; Grimsditch, M. Proc. Phys. 1989, 37, 196.

(34) Dixon, P.; Wu, L.; Nagel, S.; Williams, B. D.; Carini, J. P. Phys. Rev. Lett. 1990, 65, 1108.

(35) Roland, C. M. Macromolecules 1994, 27, 4242

(36) Ngai, K. L.; Rendell, R. W.; Plazek, J. J. Chem. Phys. 1991, 94,3018 .

(37) Adam, G.; Gibbs, J. H. J. Chem. Phys. 1965, 43, 139.

(38) Ngai, K. L. J. Non-Cryst. Solids 1991, 131-133, 80.

(39) Nozaki, R.; Mahimo, S. J. Chem. Phys. 1986, 84, 3575.

(40) Williams, G. J. Chem. Soc., Chem. Soc. Rev. 1978, 7, 89.

(41) Ngai, K. L. J. Chem. Phys. 1993, 98, 7588.

(42) Colmenero, J.; Arbe, A.; Alegria, A. Phys. Rev. Lett. 1993, 71,2603

MA9460551 